Agron. Mesoam. 30(1):63-77. Enero-abril, 2019

\title{
Antibiosis de proteínas y metabolitos en especies de Trichoderma contra aislamientos paraguayos de Macrophomina phaseolina ${ }^{1}$
}

\section{Antibiosis of proteins and metabolites of three species of Trichoderma against paraguayan isolates of Macrophomina phaseolina}

\author{
Alberto Anastacio Cubilla-Ríos ${ }^{2}$, Dani Daniel Ruíz-Díaz-Mendoza², María Cristina Romero-Rodríguez², María \\ Eugenia Flores-Giubi², Javier Enrique Barúa-Chamorro ${ }^{2}$
}

1 Recibido: 29 de agosto, 2018. Aceptado: 16 de octubre, 2018. Este trabajo formó parte de los trabajos finales de grado presentados por los dos primeros autores para optar al título de Bioquímico, y los mismos se encuentran depositados en la Biblioteca de la Facultad de Ciencias Químicas, Universidad Nacional de Asunción, Paraguay.

2 Universidad Nacional de Asunción (UNA), Facultad de Ciencias Químicas, Departamento de Química Biológica, Ruta Mcal. Estigarribia Km 11, Campus de la UNA, San Lorenzo, Paraguay. albert2cubilla@gmail.com, danidanielruizdiaz@gmail.com, mromero@rec.una.py, floresgiubi@qui.una.py, javierbarua@qui.una.py (autor para correspondencia; https://orcid.org/0000-0002-8164-3432).

\section{Resumen}

Introducción. Macrophomina phaseolina es un hongo necrotrófico de difícil control. Hongos biocontroladores, como las especies del género Trichoderma, son una alternativa para cultivos afectados por este fitopatógeno. Objetivo. El objetivo del presente trabajo fue determinar la capacidad de antibiosis de Trichoderma arundinaceum, T. brevicompactum y $T$. harzianum, contra dos aislamientos de $M$. phaseolina. Materiales y métodos. El trabajo se efectuó entre octubre de 2015 y marzo del 2016. Se utilizaron tres cepas de referencia de Trichoderma: T. arundinaceum (IBT40837), T. brevicompactum (IBT40841) y T. harzianum T34 (CECT2413) y de M. phaseolina dos aislamientos (FCQ6 y FCQ9). Se realizaron ensayos de confrontación directa, antibiosis y análisis del perfil de proteínas y metabolitos secretados por Trichoderma. Resultados. Las especies de Trichoderma empleadas inhibieron de forma significativa el crecimiento de los dos aislamientos de $M$. phaseolina en los ensayos de confrontación directa, membrana de celofán y/o membrana de diálisis. En el ensayo de confrontación directa la mayor inhibición del crecimiento de los hongos se observó a las 96 h. El hongo aislado de sésamo (Sesamun indicum L., variedad Escoba blanca), permitió la evaluación de la actividad antifúngica de las moléculas de alto y bajo peso molecular incluso hasta las $120 \mathrm{~h}$, donde T. arundinaceum mantuvo 100\% de inhibición del crecimiento; en este mismo tiempo de exposición, los hongos T. brevicompactum y T. harzianum demostraron la importancia de las moléculas de alto peso molecular para el mantenimiento de la actividad antifúngica. Los resultados mostraron la diversidad de metabolitos secundarios y proteínas secretadas por las tres especies de Trichoderma. Conclusión. Este trabajo constituye la primera descripción de la actividad antifúngica de T. arundinaceum y T. brevicompactum contra M. phaseolina, y además, se destaca el potencial de hongos aislados de suelo nativo como alternativa biológica para el control de hongos fitopatógenos de importancia agrícola.

Palabras clave: antagonismo, control biológico, hongos patógenos, micotoxinas, podredumbre carbonosa.

\begin{abstract}
Introduction. Macrophomina phaseolina is a necrotrophic fungus that is difficult to control. Biocontrol agents, like the species of the genus Trichoderma, are an alternative for the management of crop diseases caused by this


plant-pathogen. Objective. The objective of the present work was to determine the antibiosis capacity of Trichoderma arundinaceum, T. brevicompactum and T. harzianum, against two isolates of M. phaseolina. Materials and methods. Experiments were carried out from October 2015 to March 2016. Three reference strains of Trichoderma were used: T. arundinaceum (IBT40837), T. brevicompactum (IBT40841) and T. harzianum T34 (CECT2413); and two isolates of M. phaseolina (FCQ6 and FCQ9). Direct confrontation and antibiosis assays were performed, and profiling of proteins and metabolites secreted from Trichoderma. Results. The Trichoderma species significantly inhibited the growth of both $M$. phaseolina isolates in the direct confrontation assay, cellophane and/or dialysis membrane. In the direct confrontation trial, the greatest inhibition of fungal growth was observed at $96 \mathrm{~h}$. M. phaseolina isolated from sesame (Sesamum indicum L. cultivar Escoba blanca) allowed the evaluation of the antifungal activity of the molecules of high and low molecular weight even up to 120 h. T. arundinaceum maintained $100 \%$ growth inhibition in both cellophane and dialysis membrane indicating that low molecular weight metabolites were enough for complete growth inhibition of this $M$. phaseolina isolate. In contrast, T. brevicompactum and T. harzianum demonstrated the importance of high molecular weight molecules for the maintenance of antifungal activity. In addition, the complexity of secondary metabolites and proteins secreted by the three Trichoderma species was demonstrated. Conclusion. This work is the first description of the antifungal activity of T. arundinaceum and T. brevicompactum against $M$. phaseolina and also highlights the potential of fungi isolated from native soil as a biological alternative for the control of plant-pathogenic fungi of agricultural importance.

Keywords: antagonism, biological control, charcoal rot, mycotoxins, pathogenic fungi.

\section{Introducción}

Una de las enfermedades de mayor importancia en el sector agrícola es la pudrición carbonosa causada por el hongo fitopatógeno Macrophomina phaseolina (Tassi) Goid, el cual es un hongo necrotrófico, polífago y ampliamente distribuido a nivel mundial. Este hongo que es transmitido a través del suelo y semillas, subsiste en forma de microesclerocios y tiene la posibilidad de afectar a más de 500 especies de plantas, incluyendo distintas especies de granos de importancia económica como las leguminosas (Premamalini et al., 2012; Ramos et al., 2016). En los últimos años, se ha incrementado el uso indiscriminado de sustancias químicas para el control de este y otros fitopatógenos, con el consecuente impacto negativo al ambiente y a la salud de los seres humanos (Smalling et al., 2013; Peña et al., 2018). Esta problemática ha impulsado la búsqueda de nuevas estrategias que permitan reemplazar o minimizar el uso de sustancias químicas nocivas en la agricultura, una de ellas es el control biológico de fitopatógenos.

Una alternativa para el control biológico de M. phaseolina, es el uso de especies del género Trichoderma, el cual agrupa hongos cosmopolitas con gran capacidad reproductiva, alta competitividad y amplia diversidad metabólica (Lopes et al., 2012). Este grupo de hongos suele aislarse generalmente del suelo, pero también se pueden encontrar en madera húmeda en descomposición y asociados a las raíces (Chaverri y Samuels, 2003; Kubicek et al., 2008), y han sido ampliamente estudiados con el fin de controlar fitopatógenos nativos del suelo (Guigón-López et al., 2010). Son capaces de competir por el espacio, los nutrientes e inhibir el crecimiento de los patógenos usando metabolitos secundarios que actúan como fungicidas (Reino et al., 2008; Vinale et al., 2014) o degradando la pared celular por acción de enzimas hidrolíticas como quitinasas y celulasas (Infante et al., 2009; Qualhato et al., 2013; Saravanakumar et al., 2017).

Elucidar la naturaleza de los agentes responsables de la antibiosis de Trichoderma, es fundamental para la comprensión de la actividad biológica observada, y podría proporcionar nuevas estrategias de control de $M$. phaseolina más amigables con el ambiente. El objetivo del presente trabajo fue determinar la capacidad de antibiosis de Trichoderma arundinaceum, T. brevicompactum y T. harzianum, contra dos aislamientos de M. phaseolina. 


\section{Materiales y métodos}

Todos los experimentos se llevaron a cabo en la Facultad de Ciencias Químicas de la Universidad Nacional de Asunción, Paraguay, en el periodo comprendido entre octubre de 2015 y marzo del 2016.

\section{Obtención, preservación y cultivo de microorganismos}

Se utilizaron tres cepas de referencia de Trichoderma: T. arundinaceum (IBT40837), T. brevicompactum (IBT40841) y T. harzianum T34 (CECT2413); todas ellas donadas por el Departamento de Química Orgánica de la Universidad de Cádiz, y que fueron conservadas en glicerol al $80 \%$ a $-20{ }^{\circ} \mathrm{C}$. De M. phaseolina, se utilizaron dos aislamientos oriundos de Paraguay: M. phaseolina FCQ6, aislado en rastrojo de soja (Glycine max L. Merr., variedad Nidera5909rr1) y M. phaseolina FCQ9, aislado de la base del tallo de sésamo (Sesamun indicum L, variedad Escoba blanca); ambos aislamientos fueron conservados en glicerol al $80 \%$ a $4{ }^{\circ} \mathrm{C}$. Los hongos pertenecen al cepario micológico del Departamento de Química Biológica de la Facultad de Ciencias Químicas, Universidad Nacional de Asunción.

Para el mantenimiento de los hongos y para los ensayos de confrontación directa y antibiosis, se utilizó el medio de cultivo agar papa dextrosa (PDA), mientras que, para el análisis del perfil de proteínas y del perfil de metabolitos, se utilizó el medio líquido caldo papa dextrosa (PDB) (Beever y Bollard, 1970).

Para la obtención del perfil de proteínas y metabolitos secundarios de Trichoderma, se inocularon dos discos de micelio de $5 \mathrm{~mm}$ de diámetro de cada hongo, en matraces Erlenmeyer conteniendo $100 \mathrm{ml}$ de medio PDB, y se incubó durante $120 \mathrm{~h}$ en condiciones estáticas, de oscuridad y a $30^{\circ} \mathrm{C}$, los análisis se realizaron por triplicado para cada especie. El control negativo fue PDB sin inocular, mantenido en las mismas condiciones que los medios inoculados con el hongo.

\section{Ensayo de confrontación directa y antibiosis}

Las tres especies de Trichoderma y los aislamientos de M. phaseolina, se sembraron y mantuvieron en medio de cultivo PDA en oscuridad a $30{ }^{\circ} \mathrm{C}$, con el fin de preparar el pre-inóculo a utilizar en las pruebas de confrontación directa. Estas pruebas se realizaron mediante la técnica de cultivo dual, para lo cual se prepararon placas con medio PDA, en las cuales se colocaron en un extremo un disco de agar de $5 \mathrm{~mm}$ de diámetro con micelio del fitopatógeno (M. phaseolina), y en el extremo opuesto otro disco de $5 \mathrm{~mm}$ con micelio del antagonista (T. arundinaceum, $T$. brevicompactum o T. harzianum,); como controles, en placas separadas se sembró un disco de micelio de cada hongo (Figura 1A y 1B). Todas las placas se incubaron a $30^{\circ} \mathrm{C}$ en oscuridad por cinco días, tiempo después del cual se hicieron mediciones del crecimiento radial cada $24 \mathrm{~h}$, para posteriormente obtener el porcentaje de inhibición de crecimiento radial (PIC) con respecto al control. Todos los experimentos se realizaron por triplicado.

Para las pruebas de antibiosis se sembraron discos de micelio de PDA de $5 \mathrm{~mm}$ de Trichoderma en el centro de placas de PDA de $7 \mathrm{~cm}$ de diámetro, sobre membranas de diálisis (corte de $12 \mathrm{kDa}$ ) o sobre celofán, para permitir la difusión de moléculas secretadas por Trichoderma al medio de cultivo. La membrana de diálisis solamente permite la difusión de moléculas de bajo peso molecular (menor o igual a $12 \mathrm{kDa}$ ), mientras que, la membrana de celofán permite el paso de todas las moléculas secretadas por el hongo. Luego de $48 \mathrm{~h}$ de incubación a $30{ }^{\circ} \mathrm{C}$ en oscuridad, se removieron las membranas de las placas sobre el medio de cultivo y se colocaron discos de micelio de $5 \mathrm{~mm}$ de diámetro de las cepas de M. phaseolina. Al mismo tiempo, como control, los dos aislamientos del patógeno se sembraron en medio PDA. En las placas se midió el diámetro de crecimiento cada 24 h durante 96 h, 

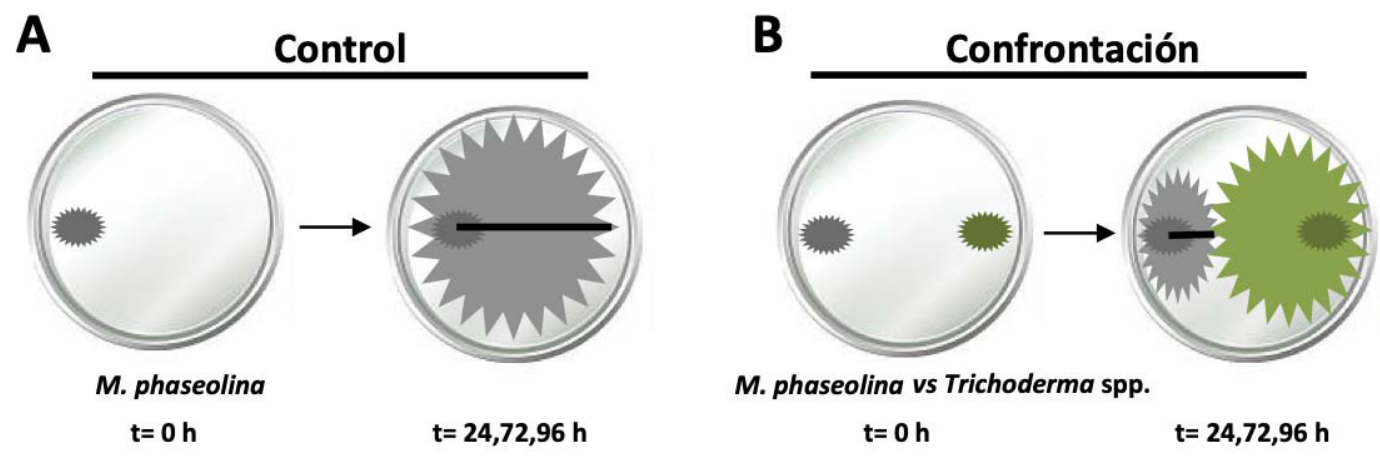

Figura 1. Ensayo de confrontación directa antagonista-patógeno de tres especies de Trichoderma contra M. phaseolina. A y B Esquema del experimento de confrontación directa del control y para la confrontación, respectivamente. Universidad Nacional de Asunción, Paraguay. 2015.

Figure 1. Direct confrontation assay antagonist-pathogen of three species of Trichoderma against M. phaseolina. A and B Scheme of the experiment of direct confrontation for the control and for the confrontation, respectively. Universidad Nacional de Asunción, Paraguay. 2015.

y los resultados se expresaron como porcentaje de inhibición del crecimiento (PIC) de las cepas de M. phaseolina por las especies de Trichoderma. El ensayo para cada aislamiento se evaluó por triplicado.

\section{Análisis estadístico}

Se utilizó un diseño experimental completamente al azar con tres repeticiones por tratamiento, los resultados se analizaron con el paquete estadístico GraphPad Prism (Motulsky, 1999). Los porcentajes de inhibición obtenidos en cada enfrentamiento en el ensayo de confrontación directa, se compararon mediante un análisis de varianza (ANOVA), y la comparación entre las medias se estimó mediante la prueba de rangos de Tukey $(\mathrm{p}=0,05)$. En el ensayo de antibiosis sobre membrana, los promedios de los PIC se compararon con la prueba de $t$ de Student. Se consideraron diferencias significativas con un $95 \%$ de confianza $\mathrm{p}<0,05$.

\section{Perfil de proteínas de Trichoderma}

Para la precipitación de proteínas secretadas por Trichoderma, el filtrado de cada cultivo se separó del micelio mediante filtración al vacío, se congeló y se liofilizó. El liofilizado fue resuspendido con una solución de solubilización [urea $8 \mathrm{M}$, tiourea $2 \mathrm{M}$ y tritón X-100 al 0,05\% (v/v)]. Posteriormente, el extracto proteico del liofilizado se concentró mediante el método de precipitación metanol-cloroformo-agua (Wessel y Flügge, 1984) y el precipitado resultante se resuspendió con solución de solubilización. Las proteínas fueron cuantificadas mediante el método descrito por Bradford (Bradford, 1976).

Veinte microgramos del concentrado de las proteínas secretadas se separaron mediante electroforesis unidimensional en geles de poliacrilamida al 12\% (Laemmli, 1970), tiñendo los geles con azul de Coomassie R-250 (Simpson, 2007). Los geles se digitalizaron mediante un fotodocumentador, y los perfiles proteicos de las diferentes especies de Trichoderma se analizaron mediante análisis de imagen. Los valores arbitrarios de intensidad de las bandas detectadas en las diferentes especies, se normalizaron respecto al total de proteínas de cada carril; el análisis comparativo de los perfiles se realizó con las bandas presentes en tres réplicas biológicas. 


\section{Obtención de los extractos orgánicos y análisis de metabolitos secundarios de Trichoderma}

Los extractos orgánicos se obtuvieron mediante la separación del micelio, a partir del medio de cultivo líquido, mediante filtración al vacío. El filtrado se extrajo exhaustivamente con acetato de etilo; las fracciones orgánicas se secaron con $\mathrm{Na}_{2} \mathrm{SO}_{4}$ anhidro, se filtraron y luego se evaporaron hasta sequedad en rotavapor. Los pesos de los extractos orgánicos obtenidos, se registraron y analizaron por cromatografía en capa fina (CCF) y cromatografía líquida de ultra eficacia (UPLC).

Para el análisis por CCF, los extractos se resuspendieron en acetato de etilo y se colocaron en cromatofolios de gel de sílice $60 \mathrm{~F}_{254}(0,20 \mathrm{~mm}$ de grosor, 4 × $6 \mathrm{~cm})$ utilizados como fase estacionaria; el hexano: acetato de etilo (1:1) se utilizaron como fase móvil. Las placas fueron visualizadas bajo luz ultravioleta a $254 \mathrm{~nm}$ y $366 \mathrm{~nm}, \mathrm{y}$ fueron expuestas a los reveladores químicos de coloración óleum, vainillina y anisaldehído.

Otra fracción de los extractos orgánicos obtenidos se disolvió en acetonitrilo (ACN) a una concentración de 5 $\mathrm{mg} / \mathrm{ml}$, se filtró con filtros de PTFE de 0,2 $\mu \mathrm{m}$, y luego se analizó por UPLC acoplado a un detector de masas en tándem (MS/MS); como fase móvil se utilizó una mezcla de agua, con 0,1\% de ácido fórmico y 0,5\% de amoníaco, más metanol en gradiente de concentración de 0 a $100 \%$ de metanol. $10 \mu \mathrm{l}$ de la muestra se inyectaron de forma automática en una columna C-18 (1,7 micras y $5 \mathrm{~cm}$ x $2,1 \mathrm{~mm})$ y se corrieron por $5 \mathrm{~min}$ a un flujo de $0,4 \mathrm{ml} / \mathrm{min}$.

\section{Resultados}

\section{Confrontación directa de Trichoderma contra M.phaseolina}

En los ensayos de confrontación se observó que las tres cepas de Trichoderma inhibieron el crecimiento de ambos aislados de M. phaseolina (Figura 2).

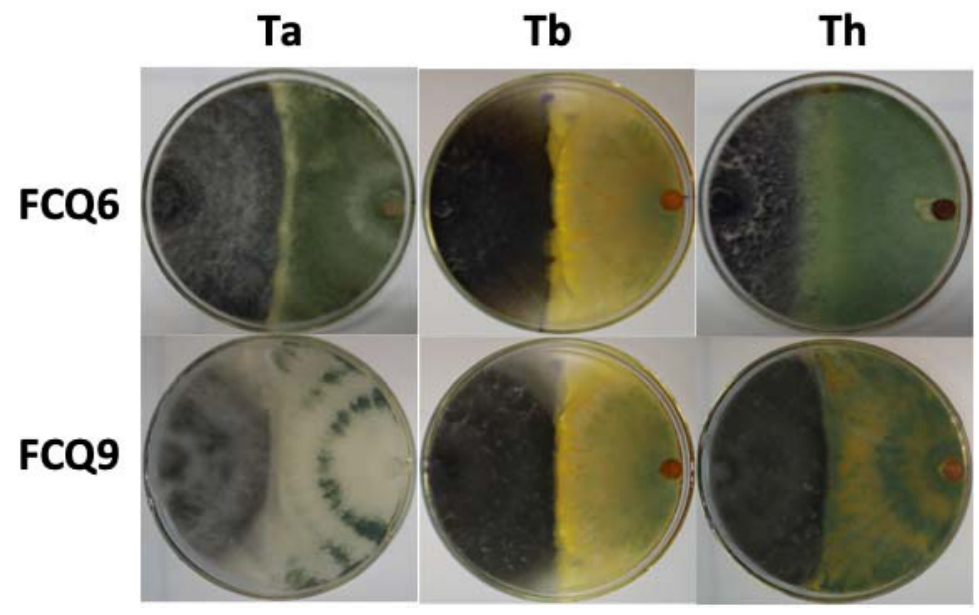

Figura 2. Ensayo de confrontación directa antagonista-patógeno de tres especies de Trichoderma contra M. phaseolina. Imágenes representativas del ensayo de confrontación directa a las $96 \mathrm{~h}$ de interacción de T. arundinaceum (Ta); T. brevicompactum (Tb) y T. harzianum (Th) con M. phaseolina aislada de soja (FCQ6) y de sésamo (FCQ9). Facultad de Ciencias Químicas, Universidad Nacional de Asunción, Paraguay. 2015.

Figure 2. Direct confrontation assay antagonist-pathogen of three species of Trichoderma against M. phaseolina. Representative images of the direct confrontation assay at $96 \mathrm{~h}$ of interaction of T. arundinaceum (Ta); T. brevicompactum (Tb) and $T$. harzianum (Th) with M. phaseolina isolated from soy (FCQ6) and from sesame (FCQ9). Facultad de Ciencias Químicas, Universidad Nacional de Asunción, Paraguay. 2015. 
En todos los casos, la mayor inhibición del crecimiento de los hongos se observó a las 96 h. El aislamiento obtenido a partir de sésamo (FCQ9) mostró mayor sensibilidad que el aislamiento de soja (FCQ6), lo que se manifestó a las 72 h cuando se los enfrentó a $T$. harzianum, y a las 72 h y 96 h cuando se los enfrentó a $T$. arundinaceum. En general, los resultados obtenidos del porcentaje de inhibición de crecimiento (PIC) presentaron diferencias estadísticamente significativas para todos los antagonistas y patógenos ensayados (Cuadro 1).

Cuadro 1. Porcentaje de inhibición del crecimiento de M. phaseolina FCQ6 y FCQ9 en confrontación directa con T. arundinaceum, T. brevicompactum and T. harzianum. Facultad de Ciencias Químicas, Universidad Nacional de Asunción, Paraguay. 2015.

Table 1. Percentage of growth inhibition of M. phaseolina FCQ6 and FCQ9 in direct confrontation assay with T. arundinaceum, T. brevicompactum and T. harzianum. Facultad de Ciencias Químicas, Universidad Nacional de Asunción, Paraguay. 2015.

\begin{tabular}{|c|c|c|c|c|c|c|c|c|c|}
\hline & \multicolumn{9}{|c|}{$*($ PIC \%) } \\
\hline & \multicolumn{3}{|c|}{ T. arundinaceum } & \multicolumn{3}{|c|}{ T. brevicompactum } & \multicolumn{3}{|c|}{ T. harzianum } \\
\hline & $48 \mathrm{~h}$ & $72 \mathrm{~h}$ & $96 \mathrm{~h}$ & $48 \mathrm{~h}$ & $72 \mathrm{~h}$ & $96 \mathrm{~h}$ & $48 \mathrm{~h}$ & $72 \mathrm{~h}$ & $96 \mathrm{~h}$ \\
\hline FCQ6 & $10 \pm 3,0^{\mathrm{a}}$ & $12,0 \pm 3,4^{\mathrm{a}}$ & $35,0 \pm 3,4^{\mathrm{b}}$ & $8,4 \pm 2,6^{\mathrm{a}}$ & $31,5 \pm 3,6^{\mathrm{b}}$ & $46,8 \pm 3,1^{\mathrm{c}}$ & $5,9 \pm 2,1^{\mathrm{a}}$ & $20,9 \pm 4,6^{b}$ & $55,6 \pm 1,6^{\mathrm{d}}$ \\
\hline FCQ9 & $9,4 \pm 5,6^{\mathrm{a}}$ & $26,1 \pm 3,0^{\mathrm{b}}$ & $49,5 \pm 1,6^{\mathrm{c}}$ & $5,0 \pm 2,0^{\mathrm{a}}$ & $30,6 \pm 8^{b}$ & $47,3 \pm 3,0^{\mathrm{c}}$ & $7,9 \pm 1,8^{\mathrm{a}}$ & $31,4 \pm 3,7^{\mathrm{c}}$ & $52,8 \pm 1,2^{\mathrm{d}}$ \\
\hline
\end{tabular}

*PIC: [(C-T) / C] X 100, PIC: porcentaje de inhibición de crecimiento, C: crecimiento del control, T: crecimiento en la confrontación. / *PIC: [(C-T) / C] X 100, PIC: percentage of growth inhibition, C: growth of control, T: growth in confrontation.

Nota: los resultados son expresados como valores de medias / Note: the results are expressed as mean values.

Letras iguales no muestran diferencias significativas entre sí ( $\mathrm{p}<0,05$ ANOVA y $\mathrm{p}<0,05$ Tukey) / Equal letters do not show significant differences between them $(\mathrm{p}<0.05$ ANOVA y $\mathrm{p}<0.05$ Tukey.

El análisis estadístico fue realizado independientemente para cada especie de Trichoderma / Statistical analysis was performed independently for each species of Trichoderma.

Inhibición de crecimiento de M. phaseolina por moléculas de alto y bajo peso molecular secretados por Trichoderma

Tanto para el experimento con membrana de celofán como con membrana de diálisis, a las $72 \mathrm{~h}$ las tres cepas de Trichoderma inhibieron por completo el crecimiento de ambos aislados de M. phaseolina FCQ6 y FCQ9 ( $\mathrm{PIC}=100 \%)$; tiempo en el cual el control de crecimiento de FCQ6 llenó la placa, finalizando el experimento para este aislamiento. Considerando que, a las 72 h, en la placa control de M. phaseolina FCQ9 aún se disponía de espacio para continuar con su crecimiento, se siguió el ensayo hasta las 120 h, cuando el hongo control llegó a su máximo crecimiento. En todos los casos hubo inhibición del crecimiento de M. phaseolina FCQ9. Tanto T. brevicompactum como T. harzianum, mostraron inhibición total de crecimiento en el ensayo sobre celofán, pero mostraron una inhibición significativamente más baja en los experimentos sobre membrana de diálisis. $T$. arundinaceum inhibió el 100\% del crecimiento del fitopatógeno en ambos experimentos (Cuadro 2).

\section{Perfil comparativo de proteínas secretadas por las especies de Trichoderma}

Las proteínas que fueron separadas en el gel de poliacrilamida, arrojaron los perfiles proteicos de las diferentes especies de Trichoderma (Figura 3). Para T. arundinaceum se detectaron un total de diecisiete bandas, para $T$. brevicompactum nueve y para $T$. harzianum once. En el carril correspondiente a los controles, se detectaron seis bandas que corresponden a proteínas propias del medio de cultivo utilizado, cuya relación de frente coincide con 
Cuadro 2. Porcentaje de inhibición del crecimiento de M. phaseolina FCQ9 por moléculas secretadas de T. arundinaceum, T. brevicompactum y T. harzianum crecidas sobre membranas de celofán o diálisis por 120 h. Facultad de Ciencias Químicas, Universidad Nacional de Asunción, Paraguay. 2016.

Table 2. Percentage of growth inhibition of M. phaseolina FCQ9 by secreted molecules of T. arundinaceum, T. brevicompactum and T. harzianum grown on cellophane or dialysis membranes for $120 \mathrm{~h}$. Facultad de Ciencias Químicas, Universidad Nacional de Asunción, Paraguay. 2016.

\begin{tabular}{ccc}
\hline Biocontroladores & \multicolumn{2}{c}{ Macrophomina phaseolina FCQ9 } \\
\cline { 2 - 3 } & Celofán & Diálisis \\
\hline T. arundinaceum & $100 \pm 0^{\mathrm{a}}$ & $100 \pm 0^{\mathrm{a}}$ \\
T. brevicompactum & $100 \pm 0^{\mathrm{a}}$ & $78,7 \pm 1,4^{\mathrm{b}}$ \\
T. harzianum & $100 \pm 0^{\mathrm{a}}$ & $77,8 \pm 1,8^{\mathrm{b}}$ \\
\hline
\end{tabular}

Nota: los resultados son expresados como valores de medias + DE / Note: the results are expressed as mean values + DE.

Para cada fila, los valores seguidos por superíndices diferentes son significativamente diferentes (t-student, $\mathrm{p}<0,01) /$ For each row, the values followed by different superscripts are significantly different ( $t$-student, $\mathrm{p}<0.01)$.

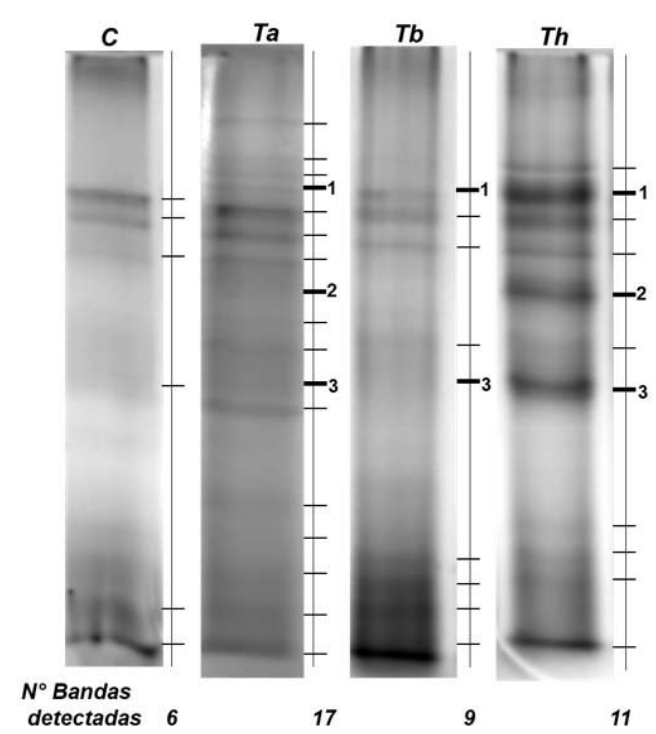

Figura 3. Perfil electroforético de proteínas secretadas por tres especies de Trichoderma $. \mathrm{C}=$ control, $\mathrm{Ta}=T$. arundinaceum, $\mathrm{Tb}=T$. brevicompactum y $\mathrm{Th}=T$. harzianum. En el lado derecho de cada gel se muestra el patrón del perfil proteico para cada especie. Abajo se indica el número de bandas detectadas en cada una de las especies de Trichoderma analizada. Facultad de Ciencias Químicas, Universidad Nacional de Asunción, Paraguay. 2016.

Figure 3. Representative electrophoretic protein profile of the different species of Trichoderma. $\mathrm{C}=$ control; $\mathrm{Ta}=$ T. arundinaceum $\mathrm{Tb}=T$. brevicompactum and $\mathrm{Th}=T$. harzianum. On the right of each gel the protein profile pattern for each species analyzed is shown. Below, the number of bands detected in each of the Trichoderma species analyzed are shown. Facultad de Ciencias Químicas, Universidad Nacional de Asunción, Paraguay. 2016.

bandas del perfil proteico de los hongos en estudio. En el patrón de las bandas para cada especie se detectaron bandas comunes y diferenciales, es decir, bandas presentes en una o dos especies analizadas (Figura 3).

En el perfil de T. harzianum se detectaron tres bandas de mayor intensidad (Figura 3 bandas 1-3). Las bandas 1 y 3 fueron detectadas en las tres especies, siendo más intensa en $T$. harzianum, mientras que la banda 2 solo se detectó en los perfiles de T. arundinaceum y T. harzianum. La banda 3 también fue detectada en el control. 


\section{Análisis cromatográfico de los extractos orgánicos de Trichoderma}

$\mathrm{Al}$ exponer las placas de la CCF a luz ultravioleta (Figuras 4A y 4B), se observaron dos manchas principales de intensidades variables, con un valor de 0,48 de relación de frente (Rf) en los carriles correspondientes a $T$. harzianum y $T$. brevicompactum, pero ausentes en el carril de $T$. arundinaceum; además, se observaron dos manchas en el carril correspondiente a T. arundinaceum a $256 \mathrm{~nm}$, con $\mathrm{Rf} 0,25$ y 0,35 , de las cuales solo la primera fue visualizada a $366 \mathrm{~nm}$ con intensidad muy baja (Figuras 4A y 4B).

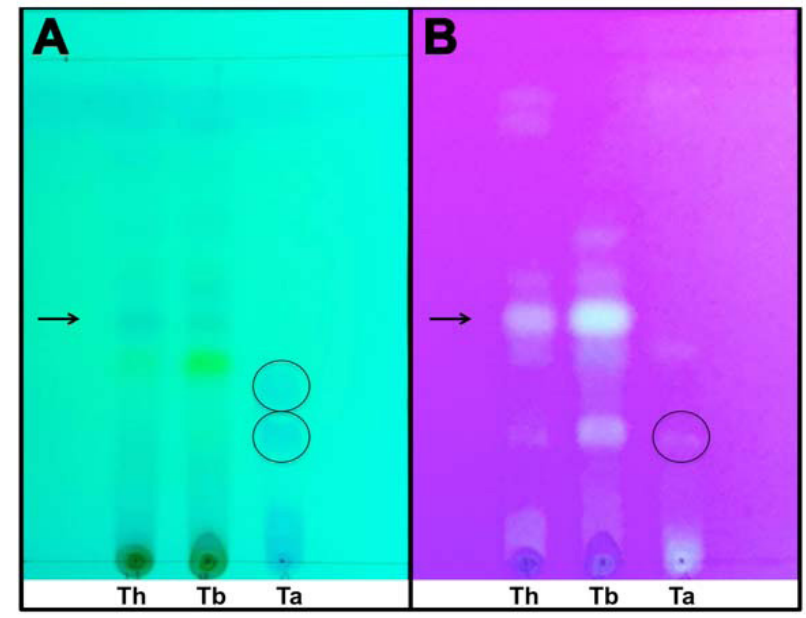

Figura 4. Cromatografía en capa delgada de los extractos orgánicos. Se utilizó como fase estacionaria gel de sílice $60 \mathrm{~F}_{254}(0,25 \mu \mathrm{m}$ de grosor) y como fase móvil hexano:acetato de etilo (1:1). Revelador físico: UV $256 \mathrm{~nm}$ (A) y UV $366 \mathrm{~nm}(\mathrm{~B})$. Th=T. harzianum, $\mathrm{Tb}=$ T. brevicompactum y $\mathrm{Ta}=T$. arundinaceum. Facultad de Ciencias Químicas, Universidad Nacional de Asunción, Paraguay. 2015

Figure 4. Thin layer chromatography of organic extracts showing the diversity of metabolites secreted into the culture medium by antagonistic fungi. Stationary phase used was silica gel $60 \mathrm{~F}_{254}(0.25 \mu \mathrm{m}$ thick $)$ and mobile phase was hexane:ethyl acetate (1:1). Physical developer: UV $256 \mathrm{~nm}$ (A) and UV $366 \mathrm{~nm}(\mathrm{~B}) . \mathrm{Th}=T$. harzianum, $\mathrm{Tb}=T$. brevicompactum y Ta= T. arundinaceum. Facultad de Ciencias Químicas, Universidad Nacional de Asunción, Paraguay. 2015.

Los reveladores químicos mostraron numerosas manchas de diferentes coloraciones (Figura 5), mostrando así la elevada diversidad de moléculas de bajo peso molecular secretadas al medio de cultivo.

Los cromatogramas de los diferentes extractos orgánicos obtenidos mediante UPLC-MS, mostraron diferencias notables entre ellos, y se emplearon filtros de selección mediante valores de relación masa:carga $(\mathrm{m} / \mathrm{z})$ de aquellos metabolitos característicos para las especies de Trichoderma. Derivado del análisis cromatográfico realizado con el extracto orgánico de $T$. harzianum, se detectó la presencia de 2-feniletanol, con $m / z=123\left(\mathrm{M}+\mathrm{H}^{+}\right)$, $\beta$-sitosterol cuyo $\mathrm{m} / \mathrm{z}$ es igual a $415\left(\mathrm{M}+\mathrm{H}^{+}\right)$, 6-pentil-2H-piran-2-ona con $\mathrm{m} / \mathrm{z} 167\left(\mathrm{M}+\mathrm{H}^{+}\right)$y 1,8-dihidroxi-3-metilantraquinona, de $m / z$ igual a $255\left(\mathrm{M}+\mathrm{H}^{+}\right)$(Figuras 6 y 7$)$.

$\mathrm{Al}$ analizar el extracto orgánico de T. arundinaceum, se detectó la presencia de 2-feniletanol con m/z igual a $123\left(\mathrm{M}+\mathrm{H}^{+}\right)$, tirosol de $\mathrm{m} / \mathrm{z}$ igual a $139\left(\mathrm{M}+\mathrm{H}^{+}\right)$y la toxina harzianum A con $\mathrm{m} / \mathrm{z}$ igual a $401\left(\mathrm{M}+\mathrm{H}^{+}\right)$(Figuras 7 y 8). Por su parte, el análisis del extracto orgánico de T. brevicompactum no dio resultados concluyentes; si bien, tanto por CCF como por UPLC-MS, se observó la producción de varios metabolitos, ninguno pudo ser identificado, debido a que no hubieron coincidencias con las masas de los metabolitos descritos previamente. 


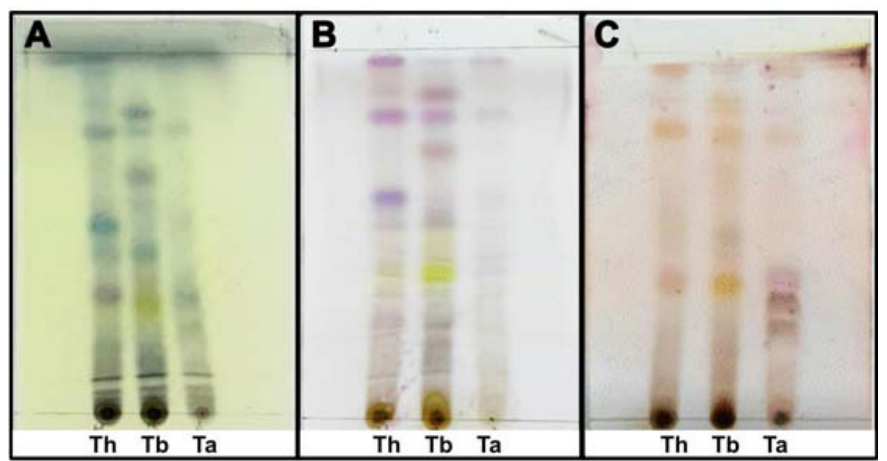

Figura 5. Cromatografía en capa delgada de los extractos orgánicos donde se observa la diversidad de metabolitos secretados al medio de cultivo por las tres especies de Trichoderma. La fase estacionaria utilizada fue gel de sílice $60 \mathrm{~F}_{254}(0,25 \mu \mathrm{m}$ de grosor) y como fase móvil se utilizó hexano:acetato de etilo (1:1). Los reveladores químicos utilizados fueron vainillina (A), anisaldehído $(\mathbf{B})$ y óleum $(\mathbf{C})$. Th= T. harzianum, $\mathrm{Tb}=T$. brevicompactum y $\mathrm{Ta}=T$. arundinaceum. Facultad de Ciencias Químicas, Universidad Nacional de Asunción, Paraguay. 2015.

Figure 5. Thin layer chromatography of organic extracts showing the diversity of metabolites secreted into the culture medium by three species of Trichoderma. Stationary phase used was silica gel $60 \mathrm{~F}_{254}(0.25 \mu \mathrm{m}$ thick); and mobile phase was hexane-ethyl acetate (1:1); Chemical developer: Vanillin $(\mathbf{A})$, Anisaldehyde $(\mathbf{B})$ and Oleum $(\mathbf{C}) . T h=T$. harzianum, $\mathrm{Tb}=$ T. brevicompactum y $\mathrm{Ta}=$ T. arundinaceum. Facultad de Ciencias Químicas, Universidad Nacional de Asunción, Paraguay. 2015.

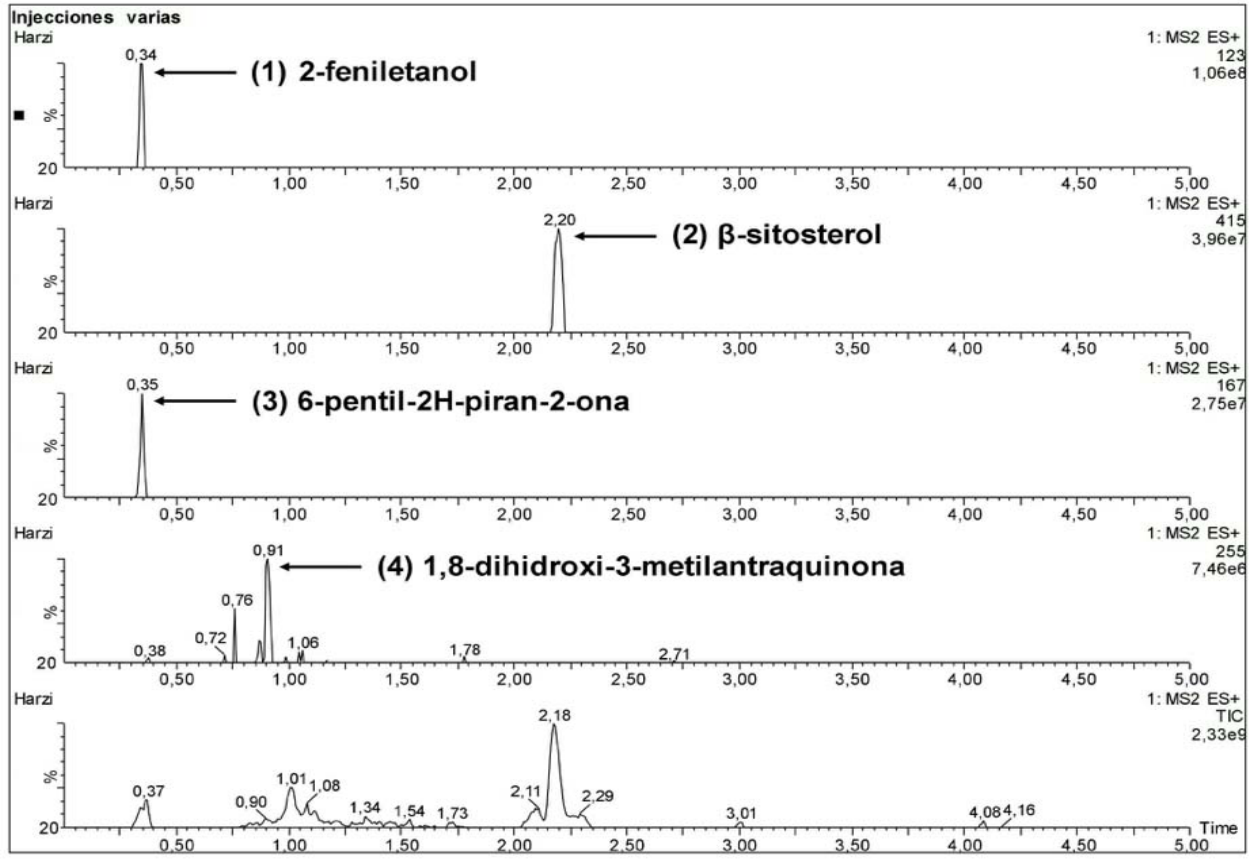

Figura 6. Cromatograma total de iones y cromatogramas de ión extraído de los extractos orgánicos de T. harzianum obtenido por UPLC-MS. En el que se observó la presencia de cuatro metabolitos: 2-feniletanol (1), $\beta$-sitosterol (2), 6-pentil-2Hpiran-2-ona (3), 1,8-dihidroxi-3-metilantraquinona (4), fase estacionaria: columna C18 de 1,7 micras; fase móvil: aguametanol en gradiente de concentración de 0 a $100 \%$ de metanol. Facultad de Ciencias Químicas, Universidad Nacional de Asunción, Paraguay. 2015.

Figure 6. Total ion current and ion chromatograms from the organic extracts of T. harzianum obtained by UPLC-MS, in which the presence of four metabolites was observed: 2-phenylethanol (1), $\beta$-sitosterol (2), 6-pentyl-2H-pyran-2-one (3), 1,8-dihydroxy3-methylanthraquinone (4), stationary phase: column C18 of 1.7 microns; mobile phase: water-methanol with gradient increased from 0 to 100\% methanol. Facultad de Ciencias Químicas, Universidad Nacional de Asunción, Paraguay. 2015. 


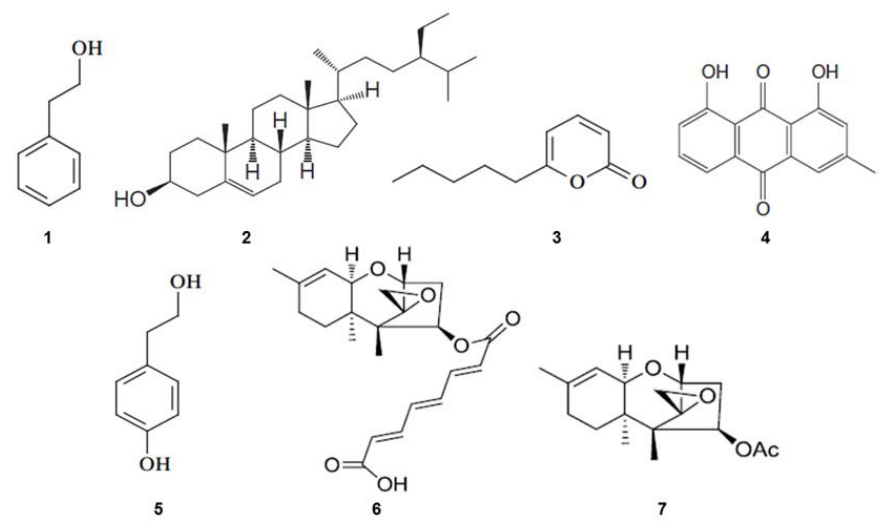

Figura 7. Estructura química de los metabolitos analizados en tres especies de Trichoderma (T. arundinaceum, T. brevicompactum y T. harzianum): 2-feniletanol (1), $\beta$-sitosterol (2), 6-pentil-2H-piran-2-ona (3), 1,8-dihidroxi-3-metilantraquinona (4), tirosol (5), harzianum A (6) y trichodermina (7). Facultad de Ciencias Químicas, Universidad Nacional de Asunción, Paraguay. 2015.

Figure 7. Chemical structure of metabolites analyzed in three species of Trichoderma (T. arundinaceum, T. brevicompactum and T. harzianum): 2-phenylethanol (1), $\beta$-sitosterol (2), 6-pentyl-2H-pyran-2-one (3), 1,8-dihydroxy-3-methylanthraquinone (4), tyrosol (5), harzianum A (6) and trichodermin (7). Facultad de Ciencias Químicas, Universidad Nacional de Asunción, Paraguay. 2015.

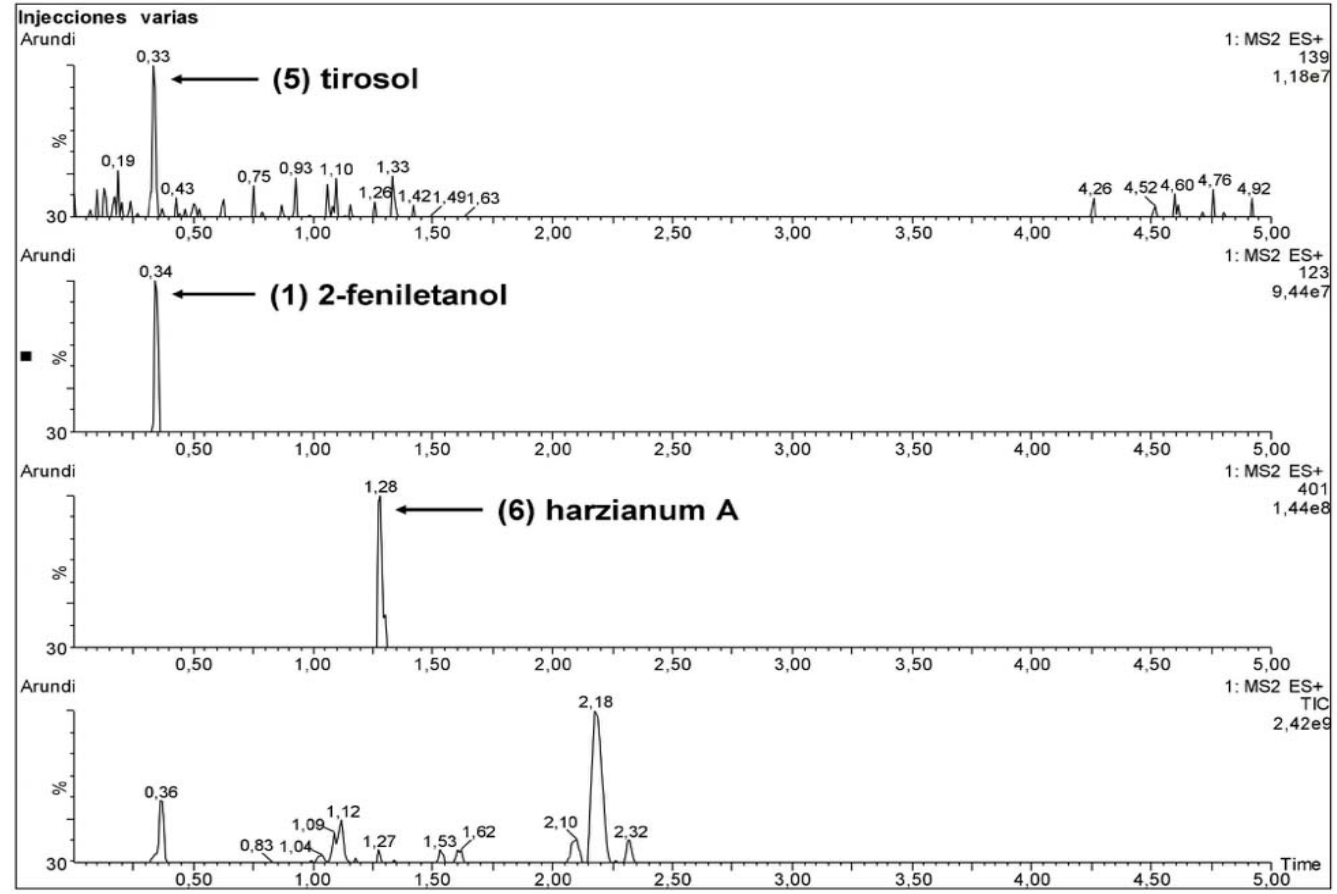

Figura 8. Cromatograma total de iones y cromatogramas de ión extraído de los extractos orgánicos de T. arundinaceum obtenida por UPLC-MS, en el que se observó la presencia de tres metabolitos tirosol (5), 2-feniletanol (1), y harzianum A (6). Fase estacionaria: Columna C18 de 1,7 micras; fase móvil: agua-metanol en gradiente de concentración de 0 a $100 \%$ de metanol. Facultad de Ciencias Químicas, Universidad Nacional de Asunción, Paraguay. 2015.

Figure 8. Total ion current (TIC) and ion chromatograms from the organic extracts of T. arundinaceum obtained by UPLC-MS, in which the presence of metabolites was observed: tyrosol (5), 2-phenylethanol (1), and harzianum A (6). Stationary phase: Column C18 of 1.7 microns; mobile phase: water-methanol was gradient increased from 0 to $100 \%$ methanol. Facultad de Ciencias Químicas, Universidad Nacional de Asunción, Paraguay. 2015. 


\section{Discusión}

En general, las tres especies de Trichoderma empleadas en este trabajo inhibieron de forma significativa el crecimiento de los dos aislados de M. phaseolina en los ensayos de confrontación directa, membrana de celofán y/o membrana de diálisis.

Mediante el ensayo de confrontación directa hubo capacidad biocontroladora de las tres cepas de Trichoderma evaluadas, contra ambos aislados de M. phaseolina. Otras cepas de T. harzianum han demostrado previamente su eficacia en la inhibición de M. phaseolina (Khalili et al., 2016), sin embargo, no se encontraron referencias previas que describan el biocontrol ejercido por T. brevicompactum y T. arundinaceum contra M. phaseolina.

Con respecto a la especificidad de la interacción entre las especies de Trichoderma probadas y M. phaseolina, se observó que el aislamiento proveniente de sésamo (FCQ9) fue el más sensible al enfrentarse a T. arundinaceum; dicho efecto fue más evidente a las $96 \mathrm{~h}$. Se ha descrito que la variabilidad genética de M. phaseolina está relacionada con el origen geográfico y del hospedero, condición que puede afectar tanto a la morfología como a los procesos biológicos del fitopatógeno (Baird et al., 2010; Arias et al., 2011; Mahdizadeh et al., 2011; Khan et al., 2017), lo que explica la distinta respuesta observada ante una misma situación de estrés, como pudo ser la exposición a agentes de biocontrol.

La evaluación de la naturaleza química de las moléculas responsables de la actividad antifúngica, reveló que las moléculas de alto y bajo peso molecular secretados por las tres especies de Trichoderma mantuvieron 100\% de PIC hasta las $72 \mathrm{~h}$ de exposición para ambos aislados de M. phaseolina; de esta manera, la presencia de ambos tipos de moléculas secretadas al medio de cultivo se asociaron con mayor antibiosis. A las $120 \mathrm{~h}$ del experimento con membranas, las moléculas de bajo peso molecular secretadas por $T$. harzianum y T. brevicompactum mostraron una menor actividad antifúngica sobre el aislado de sésamo, por lo que, puede inferirse que las moléculas de alto peso molecular secretadas por Trichoderma al medio fueron un factor importante en su actividad antifúngica.

Hubo diferente complejidad del perfil proteico de las proteínas secretadas por las tres especies de Trichoderma, y según los resultados de actividad antifúngica obtenidos, las proteínas secretadas por $T$. harzianum y $T$. brevicompactum, tuvieron un rol importante en la inhibición del crecimiento del fitopatógeno M. phaseolina aislado de sésamo, considerando los resultados obtenidos a las $120 \mathrm{~h}$ en el experimento con membranas de celofán y diálisis.

El análisis por CCF mostró la presencia de varios compuestos en los extractos orgánicos de Trichoderma, de los cuales podrían ser responsables de la antibiosis observada con membrana de diálisis. La actividad antifúngica de los metabolitos secretados por T. harzianum y detectados en el presente trabajo, ha sido evaluada previamente contra varios fitopatógenos, entre ellos $M$. phaseolina, y la 1,8-dihidroxi-3-metilantraquinona, es la que ha demostrado una mayor capacidad de inhibición del crecimiento fúngico (Ahluwalia et al., 2015). Las proteínas secretadas por $T$. harzianum han sido analizadas en diferentes medios de cultivo por diferentes autores (Suárez et al., 2005; Monteiro et al., 2010), y se observa un perfil similar al que se obtuvo en el presente trabajo. Por otro lado, en trabajos previos se ha confirmado la producción de proteínas con actividad hidrolítica como endoglucanas, celulasas y xilanasas (Gómez-Mendoza et al., 2014), proteasa aspártica (Suárez et al., 2005) y quitinasas (Nagy et al., 2007), enzimas involucradas en la antibiosis ejercida por Trichoderma spp. (Harman, 2006). También se ha detectado y descrito actividad de proteasas en medios de cultivos mínimos sin estímulos, en los cuales ha crecido T. harzianum (Ramada et al., 2016).

Respecto al perfil de proteínas secretadas por T. brevicompactum, se detectó un menor número de bandas, proteínas que podrían estar involucradas en la antibiosis. Se ha descrito que proteínas secretadas por $T$. brevicompactum al medio de cultivo poseen actividad $\beta-1,3-$ y $\beta-1,4-$ glucanasa, y son capaces de degradar la pared celular de fitopatógenos (Ayoubi et al., 2014); en las condiciones experimentales del presente trabajo, estas proteínas podrían estar presentes y haber sido secretadas al medio. En T. brevicompactum es característica 
la producción de la micotoxina trichodermina (Nielsen et al., 2005), sin embargo, en las condiciones de cultivo empleadas en este trabajo no se detectó su presencia.

Trichoderma arundinaceum inhibió en alto porcentaje (100\%) el crecimiento (PIC) de M. phaseolina aislado de sésamo hasta las $96 \mathrm{~h}$, en el ensayo de confrontación directa y de membranas. Resultados similares se han obtenido al evaluar el crecimiento de Botrytis cinerea Pers. y Rhizoctonia solani J.G. Kühn por cepas silvestres y mutantes de T. arundinaceum, siguiendo el mismo método (Malmierca et al., 2012). En el análisis químico del extracto de T. arundinaceum, se destacó la presencia del metabolito harzianum A, el cual ha sido previamente descrito en este hongo (Corley et al., 1994) y del que se conoce que es una potente micotoxina perteneciente a la familia de los trichotecenos (Lee et al., 2005); además, en Trichoderma spp. se ha descrito la presencia de péptidos con actividad antibiótica, cuyo peso molecular varía entre 500 y los $2200 \mathrm{Da}$ (Degenkolb et al., 2003), mismos que podrían ejercer la antibiosis junto con los metabolitos en el ensayo de membrana de diálisis.

Para T. arundinaceum, los resultados de crecimiento sobre membrana no permitieron demostrar la contribución de las proteínas secretadas y detectadas en el perfil proteico, debido a la elevada actividad antifúngica de las moléculas de bajo peso molecular secretados por el agente de biocontrol, reflejados en el PIC 100\% de los experimentos con membrana de celofán y diálisis. Estos resultados coinciden con los obtenidos por Malmierca et al. (2012) para R. solani, y contrastan con los obtenidos por los mismos autores para B. cinerea, en donde el PIC en membrana de celofán fue significativamente mayor que en diálisis, demostrando la importancia de las proteínas secretadas en la antibiosis contra B. cinerea.

\section{Conclusiones}

Las tres especies de Trichoderma empleadas inhibieron de forma significativa el crecimiento de ambos aislados de M. phaseolina. Estas especies son capaces de biosintetizar varias moléculas de alto y bajo peso molecular que están implicadas en el efecto antagónico y de antibiosis observado contra los dos aislamientos de M. phaseolina. Este trabajo constituye la primera descripción de la actividad antifúngica de T. brevicompactum y T. arundinaceum contra M. phaseolina.

\section{Agradecimientos}

Los autores expresan sus agradecimientos a las personas quienes gentilmente han donado los hongos que han sido utilizados en este trabajo. Al Dr. Isidro G. Collado del Dpto. de Química Orgánica, Facultad de Ciencias, Universidad de Cádiz, por las cepas de Trichoderma y al Dr. Pablo H. Sotelo y el Dr. Julio C. M. Iehisa del Departamento de Biotecnología, Facultad de Ciencias Químicas, Universidad Nacional de Asunción, por los aislados de M. phaseolina.

\section{Literatura citada}

Ahluwalia, V., J. Kumar, V.S. Rana, O.P. Sati, and S. Walia. 2015. Comparative evaluation of two Trichoderma harzianum strains for major secondary metabolite production and antifungal activity. Nat. Prod. Res. 29:914-920. doi:10.1080/147 86419.2014.958739

Arias, R.S., J.D. Ray, A. Mengistu, and B.E. Scheffler. 2011. Discriminating microsatellites from Macrophomina phaseolina and their potential association to biological functions. Plant Pathol. 60:709-718. doi:10.1111/j.1365-3059.2010.02421.x 
Ayoubi, N., D. Zafari, and M. Mirabolfathy. 2014. Evaluation of $\beta$-1,3-glucanase and $\beta$-1,4-glucanase enzymes production in some Trichoderma species. Arch. Phytopathol. Plant Prot. 47:1929-1941. doi:10.1080/03235408.2013.862457

Baird, R.E., P.A. Wadl, T. Allen, D. McNeill, X. Wang, J.K. Moulton, T.A. Rinehart, H.K. Abbas, T. Shier, and R.N. Trigiano. 2010. Variability of United States isolates of Macrophomina phaseolina based on simple sequence repeats and cross genus transferability to related genera within Botryosphaeriaceae. Mycopathologia 170(3):169-180. doi:10.1007/s11046-0109308-3

Beever, R., and E.G. Bollard. 1970. The nature of the stimulation of fungal growth by potato extract. J. Gen. Microbiol. 60:273279. doi:10.1099/00221287-60-2-273

Bradford, M.M. 1976. A rapid and sensitive method for the quantitation of microgram quantities of protein utilizing the principle of protein-dye binding. Anal. Biochem. 72:248-254. doi:10.1016/0003-2697(76)90527-3

Chaverri, P., and G.J. Samuels. 2003. Hypocrea/Trichoderma (Ascomycota, Hypocreales, Hypocreaceae): Species with green ascospores. Stud. Mycol. 48:1-119.

Corley, D., M. Miller-Wideman, and R. Durley. 1994. Isolation and structure of harzianum A: a new trichothecene from Trichoderma harzianum. J. Nat. Prod. 156:422-425. doi:10.1021/np50105a019

Degenkolb, T., A. Berg, W. Gams, B. Schlegel, and U. Gräfe. 2003. The occurrence of peptaibols and structurally related peptaibiotics in fungi and their mass spectrometric identification via diagnostic fragment ions. J. Pept. Sci. 9:666-678. doi: $10.1002 /$ psc.497

Gómez-Mendoza, D.P., M. Junqueira, L.H.F. Do Vale, G.B. Domont, E.X. Ferreira Filho, M.V. De-Sousa, and C.A.O. Ricart. 2014. Secretomic survey of Trichoderma harzianum grown on plant biomass substrates. J. Proteome Res. 13:1810-1822. doi:10.1021/pr400971e

Guigón-López, C., V. Guerrero-Prieto, F. Vargas-Albores, E. Carvajal-Millán, G.D. Ávila-Quezada, L. Bravo-Luna, M. Ruocco, S. Lanzuise, S. Woo, y M. Lorito. 2010. Identificación molecular de cepas nativas de Trichoderma spp. su tasa de crecimiento in vitro y antagonismo contra hongos fitopatógenos. Rev. Mex. Fitopatol. 28(2):87-96.

Harman, G.E. 2006. Overview of mechanisms and uses of Trichoderma spp. Phytopathology 96:190-194. doi:10.1094/ PHYTO-96-0190

Infante, D., B. Martínez, N. González, y Y. Reyes. 2009. Mecanismos de acción de Trichoderma frente a hongos fitopatógenos. Rev. Protección Veg. 24(1):14-21.

Khalili, E., M.A. Javed, F. Huyop, S. Rayatpanah, S. Jamshidi, and R.A. Wahab. 2016. Evaluation of Trichoderma isolates as potential biological control agent against soybean charcoal rot disease caused by Macrophomina phaseolina. Biotechnol. Biotechnol. Equip. 30:479-488. doi:10.1080/13102818.2016.1147334

Khan, A.N., F. Shair, K. Malik, Z. Hayat, M.A. Khan, F.Y. Hafeez, and M.N. Hassan. 2017. Molecular identification and genetic characterization of Macrophomina phaseolina strains causing pathogenicity on sunflower and chickpea. Front. Microbiol. 8:1309. doi:10.3389/fmicb.2017.01309.

Kubicek, C.P., M. Komon-Zelazowska, and I.S. Druzhinina. 2008. Fungal genus Hypocrea/Trichoderma: from barcodes to biodiversity. J. Zhejiang Univ. Sci. B 9:753-763. doi:10.1631/jzus.B0860015

Laemmli, U.K. 1970. Cleavage of structural proteins during the assembly of the head of bacteriophage T4. Nature 227:680-685. doi: $10.1038 / 227680 \mathrm{a} 0$

Lee, H.B., Y. Kim, H.Z. Jin, J.J. Lee, C.J. Kim, J.Y. Park, and H.S. Jung. 2005. A new Hypocrea strain producing harzianum A cytotoxic to tumour cell lines. Lett. Appl. Microbiol. 40:497-503. doi:10.1111/j.1472-765X.2005.01719.x 
Lopes, F.A.C., A.S. Steindorff, A.M. Geraldine, R.S. Brandão, V.N. Monteiro, M.L. Júnior, A.S.G. Coelho, C.J. Ulhoa, and R.N. Silva. 2012. Biochemical and metabolic profiles of Trichoderma strains isolated from common bean crops in the Brazilian Cerrado, and potential antagonism against Sclerotinia sclerotiorum. Fungal Biol. 116:815-824. doi:10.1016/j. funbio.2012.04.015

Mahdizadeh, V., N. Safaie, and E.M. Goltapeh. 2011. Diversity of Macrophomina phaseolina based on morphological and genotypic characteristics in Iran. Plant Pathol. J. 27(2):128-137. doi:10.5423/PPJ.2011.27.2.128

Malmierca, M.G., R.E. Cardoza, N.J. Alexander, S.P. McCormick, R. Hermosa, E. Monte, and S. Gutiérrez. 2012. Involvement of Trichoderma trichothecenes in the biocontrol activity and induction of plant defense-related genes. Appl. Environ. Microbiol. 78:4856-4868. doi:10.1128/AEM.00385-12

Monteiro, V.N., R. do-Nascimento-Silva, A.S. Steindorff, F.T. Costa, E.F. Noronha, C.A.O. Ricart, M.V. de-Sousa, M.H. Vainstein, and C.J. Ulhoa. 2010. New insights in Trichoderma harzianum antagonism of fungal plant pathogens by secreted protein analysis. Curr. Microbiol. 61:298-305. doi:10.1007/s00284-010-9611-8

Motulsky HJ. 1999. Analyzing data with GraphPad Prism. GraphPad Software Inc., CA, USA.

Nagy, V., V. Seidl, G. Szakacs, M. Komon-Zelazowska, C.P. Kubicek, and I.S. Druzhinina. 2007. Application of DNA bar codes for screening of industrially important fungi: The haplotype of Trichoderma harzianum sensu stricto indicates superior chitinase formation. Appl. Environ. Microbiol. 73:7048-7058. doi:10.1128/AEM.00995-07

Nielsen, K.F., T. Gräfenhan, D. Zafari, and U. Thrane. 2005. Trichothecene production by Trichoderma brevicompactum. J. Agric. Food Chem. 53:8190-8196. doi: 10.1021/jf051279b

Peña, N., A. Antón, A. Kamilaris, and P. Fantke. 2018. Modeling ecotoxicity impacts in vineyard production: addressing spatial differentiation for copper fungicides. Sci. Total Environ. 616-617:796-804. doi:10.1016/j.scitotenv.2017.10.243

Premamalini, T., B.T. Ambujavalli, R. Vijayakumar, S.V. Rajyoganandh, S. Kalpana, and A.J. Kindo. 2012. Fungal keratitis caused by Macrophomina phaseolina - A case report. Med. Mycol. Case Rep. 1(1):123-126. doi:10.1016/j. mmcr.2012.10.007

Qualhato, T.F., F.A.C. Lopes, A.S. Steindorff, R.S. Brandão, R.S.A. Jesuino, and C.J. Ulhoa. 2013. Mycoparasitism studies of Trichoderma species against three phytopathogenic fungi: Evaluation of antagonism and hydrolytic enzyme production. Biotechnol. Lett. 35:1461-1468. doi:10.1007/s10529-013-1225-3

Ramada, M.H.S., A.S. Steindorff, C. Bloch, and C.J. Ulhoa. 2016. Secretome analysis of the mycoparasitic fungus Trichoderma harzianum ALL 42 cultivated in different media supplemented with Fusarium solani cell wall or glucose. Proteomics 16:477-490. doi:10.1002/pmic.201400546

Ramos, A.M., M. Gally, G. Szapiro, T. Itzcovich, M. Carabajal, y L. Levin. 2016. Crecimiento in vitro y producción de enzimas degradadoras de pared celular vegetal de aislamientos argentinos de Macrophomina phaseolina, agente causal de la podredumbre carbonosa en maíz. Rev. Argent. Microbiol. 48:267-273. doi:10.1016/j.ram.2016.06.002

Reino, J.L., R.F. Guerrero, R. Hernández-Galán, and I.G. Collado. 2008. Secondary metabolites from species of the biocontrol agent Trichoderma. Phytochem. Rev. 7:89-123. doi:10.1007/s11101-006-9032-2

Saravanakumar, K., Y. Li, C. Yu, Q.Q. Wang, M. Wang, J. Sun, J.X. Gao, and J. Chen. 2017. Effect of Trichoderma harzianum on maize rhizosphere microbiome and biocontrol of Fusarium Stalk rot. Sci. Rep. 7:1-13. doi:10.1038/s41598-017-01680-w

Simpson, R.J. 2007. Staining proteins in gels with coomassie blue. CSH Protoc. 2007:pdb.prot4719. doi:10.1101/pdb.prot4719

Smalling, K.L., K.M. Kuivila, J.L. Orlando, B.M. Phillips, B.S. Anderson, K. Siegler, J.W. Hunt, and M. Hamilton. 2013. Environmental fate of fungicides and other current-use pesticides in a central California estuary. Mar. Pollut. Bull. 73:144153. doi:10.1016/j.marpolbul.2013.05.028 
Suárez, M.B., L. Sanz, M.I. Chamorro, M. Rey, F.J. González, A. Llobell, and E. Monte. 2005. Proteomic analysis of secreted proteins from Trichoderma harzianum: Identification of a fungal cell wall-induced aspartic protease. Fungal Genet. Biol. 42:924-934. doi:10.1016/j.fgb.2005.08.002

Vinale, F., K. Sivasithamparam, E.L. Ghisalberti, S.L. Woo, M. Nigro, R. Marra, N. Lombardi, A. Pascale, M. Ruocco, S. Lanzuise, G. Manganiello, and M. Lorito. 2014. Trichoderma secondary metabolites active on plants and fungal pathogens. Open Mycol. J. 8:127-139. doi:10.2174/1874437001408010127.

Wessel, D., and U.I. Flügge. 1984. A method for the quantitative recovery of protein in dilute solution in the presence of detergents and lipids. Anal. Biochem. 138:141-143. doi:10.1016/0003-2697(84)90782-6. 